\title{
Beiträge zur Kenntnis der Chronologie des "Jüngeren Lösses" in der Dobrudscha (Rumänische Volksrepublik)
}

\author{
Von Petre Samson und Constantin Rădulescu, Bukarest \\ Mit 1 Tabelle
}

$\mathrm{Z}$ us a m m e n f assung. Beim Studium der Lößablagerungen von Poarta Albă, in den Höhlen „La Adam", Bordeiul de Piatră“ und „Adădpostul Rîndunelelor" war es möglich, das Vorhandensein von zwei hauptsächlichen Ablagerungsperioden in der Dobrudscha festzustellen und zwar: Jüngerer Löß I, der eine arktische Fauna und Hoch-Moustier-Industrie aufweist und Jüngerer Löß II, welcher in zwei Etappen abgelagert wurde. Jüngerer Löß IIa und IIb zeigen die größte Häufigkeit an arktischer Fauna und Steppentieren, der eine in Verbindung mit der MittelAurignac-Industrie, der andere mit der Gravette-Industrie. Zwischen den beiden Schichten des Jüngeren Löß II befindet sich ein interstadiales Niveau, das Vistorna-Interstadial, welches für diese, von der gemäßigten Fauna beherrschten Gegend charakteristisch ist und Instrumente aus der Prägravette-Industrie geliefert hat.

In der Dobrudscha bildete sich während des Oberen Pleistozäns eine Waldsteppen-Landschaft, was durch die Anwesenheit der Vertreter der Steppen- und Waldfauna bewiesen ist; die Anwesenheit der arktischen Tiere ist in dieser Gegend nur auf deren Winterwanderung zurückzuführen.

R é s u m é. En étudiant les dépôts de loess de Poarta Albă et des grottes "La Adam", "Bordeiul de Piatră" et "Adăpostul Rîndunelelor", il a été possible de préciser l'existence de deux périodes principales de sédimentation en Dobroudscha, représentées par le loess récent I quai a fourni une faune arctique et une industrie moustiérienne supérieure et le loess récent II, déposé en deux étapes: le loess récent IIa et IIb qui surprennent les maxima de fréquence des espèces arctiques et de steppes, associés l'un avec une industrie aurignacienne moyenne et l'autre avec une industrie gravettienne. Entre les deux couches de loess récent II s'interpose un niveau interstadial L'INTERSTADE VISTORNA - propre à cette région, dominé par une faune a cachet tempéré et qui a livré des instruments prégravettiens. En Dobroudscha, pendant le Pléistocene supérieur, s'est établi un paysage de sylvo-steppe, témoin l'existence, en même temps, des espèces steppiques et de forêt; la présence des éléments arctiques, dans la région, est dûe seulement à leurs migrations d'hiver.

Die Paläontologische Abteilung des Institutes für Speologie „E. Racoviţa“ in Bukarest hat unter der Leitung des Univ.-Prof. Dr. C. Motaş im Jahre 1956 paläontologische Untersuchungen in den Ablagerungen der Höhlen in der Dobrudscha unternommen, die in den wissenschaftlichen Kreisen durch die Arbeiten der Frau Univ.-Prof. Dr. M. DumITREscu und des Herrn Dr. T. Orghidan bekannt geworden sind.

Unsere Beobachtungen beziehen sich einerseits auf die Ablagerungen in den Höhlen „La Adam“, „Bordeiul de Piatră“ (Steinhütte) und „Adăpostul Rîndunelelor“ (Schwalbenfelsnische), welche sich im Flußbecken des Casimcea befinden, andererseits auf dem Löß der Ziegelfabrik in Poarta Albăํ).

Die Füllablagerung der Höhle „La Adam" gibt in unserem Lande das vollständigste Schichtenprofil über das Obere Pleistozän und umfaßt den Abschnitt vom Ende der Interglazialzeit Riß/Würm bis jetzt. In diesem Schichtenprofil, welches bei dem heutigen Stand der Studien in einer Tiefe von $9 \mathrm{~m}$ und einer Länge von $40 \mathrm{~m}$ untersucht ist, bildet der Löß drei Hauptschichten, welche in den verschiedenen Perioden der letzten Eiszeit abgelagert wurden.

Die Ơffnung der Höhle ist ziemlich klein, und der Löß hat sich anfänglich nur im Raume des Einganges abgesetzt. Erst nach dem ersten Interstadial des Mittelwürms, als die Decke der Höhle teilweise einstürzte, war die Ablagerung auch im Inneren der Höhle möglich, ohne aber die Stärke zu erreichen, welche sich im Eingang der Höhle gebildet hat.

1) Die Arbeiten wurden unter der Mitwirkung des Museums für Altertumsforschung in Constanţa durchgeführt. 
Infolge der kleinen Abmessungen und der großen Offnungen bei der Höhle „Bordeiul de Piatră“ und bei der „Adăpostul Rîndunelelor" hat sich der Löß auf der ganzen Fläche der Böden abgesetzt. Während bei der "Adăpostul Rîndunelelor" die Ablagerung in der ganzen Stärke nur aus Löß besteht, finden wir den Löß bei der „Bordeiul de Piatră“ nur in der Zusammensetzung der untersten Schicht, welche durch wiederholte Uberschwemmungen ausgewaschen wurde und daher in ihrem oberen Teil nur aus feinem olivgrünem Sand besteht.

Der Löß in Poarta Albă, der eine Stärke von $15 \mathrm{~m}$ aufweist, hat uns Überreste der Fauna in dem untersten Teil unter $12 \mathrm{~m}$ Tiefe geliefert. Wir haben keine charakreristischen Schichtungen in dieser Ablagerung feststellen können.

$\mathrm{Da}$ die untersuchten Ablagerungen sich während der Würm-Eiszeit gebildet haben, stellen sie einen "Jüngeren Löß“ dar.

Das Problem, welches hier zu klären ist, behandelt die einzelnen Phasen der Ablagerung des Lösses, und unsere, auf dem Schichtenverband, den Tiergesellschaften sowie auf den Spuren der menschlichen Industrie beruhenden Studien haben gezeigt, daß es in der Dobrudscha drei Zeitabschnitte gab, welche dem "Jüngeren Löß I“, dem „Jüngeren Löß II $a$ “ und dem „Jüngeren Löß II b“ entsprechen.

\section{Jüngerer Löß I}

Die Lößablagerung im Frühwürm ist in der Höhle „La Adam“ durch zwei Wohnstätten-Niveaus vertreten; die Stärke beträgt $0,80 \mathrm{~m}$ und sie befinden sich in einer 'Tiefe, die zwischen $2 \mathrm{~m}$ beim Eingang und 4,50 $\mathrm{m}$ in der Mitte der Höhle liegt.

Was die Fauna anbetrifft, sind die Schichten durch die zahlreiche Anwesenheit des Ursus spelaeus in Gemeinschaft mit dem Mammonteus primigenius und Coelodonta antiquitatis gekennzeichnet, welche typische Vertreter des Dobrudschaer Hoch- und SpätMoustier sowie des Früh-Aurignac sind. Eine arktische Prägung zeigt das Erscheinen des Alopex lagopus, Rangifer tarandus und der Nyctea sp. Ebenso fehlen nicht die Steppenformen, wie Saiga tatarica und Equus (Asinus) bydruntinus. Neben dieser Tiergesellschaft, welche eine Kälteperiode anzeigt, bestehen noch aus der Interglazialzeit die Tierarten, welche an bewaldete Gegenden gebunden sind, wie der Rothirsch und der Rie se $\mathrm{nh}$ irsch. Die genannte Fauna unterscheidet die Moustier-Schichten von den tiefer unten gelegenen, welche eine temperierte Fauna aufweisen (Capreolus capreolus, Cervus elaphus, Megaceros). Diese tieferen Schichten sind von den Moustier-Schichten durch eine vollkommen sterile Schicht sowohl faunistisch als archäologisch getrennt.

Die menschliche Industrie zeigt sich in den Steinwerkzeugen, die in der Technik des Oberen Moustier gearbeitet sind und in den beiden übereinanderliegenden Herdniveaus gefunden wurden. Besonders bemerkenswert sind die Absplisse und Kratzer, die aus einem Silex minderwertiger Qualität gehauen sind. Dieser Silex stammt in der Mehrzahl der Fälle von Silikatkonkretionen des Rauracien-Kalksteins, der in dieser Gegend vorkommt.

Der gleichen Periode müssen wir den untersten Teil des Lösses in Poarta Albă zuschreiben, der fossile Knochen des Mammonteus primigenius, Coelodonta antiquitatis, Rangifer tarandus, Megaceros giganteus, Bos primigenius, Equus caballus und Equus caballus - große Form - geliefert hat.

Eine gemeinsame Charakteristik des moustierzeitlichen fossilen Materiales aus den Fundstellen „La Adam“ und „Poarta Albă“ ist die tiefschwarze Färbung und die starke Verkrustung infolge des Absatzes von Kalziumkarbonat.

Die untere, bei $0,90 \mathrm{~m}$ Tiefe befindliche, einen halben Meter dicke Schicht der Ablagerung in der Höhle „Bordeiul de Piatră“ stimmt infolge der großen Häufigkeit des Ursus spelaeus und der Anwesenheit der Steppenformen wie Saiga tatarica und Bison priscus 
mit den Hoch-Moustier-Schichten der Höhle „La Adam“ überein, der sie auch in der schwarzen Farbe der Knochen ähnelt. Diese Tatsache ist auch durch das Bestehen einer Spät-Moustier-Industrie in der darüberliegenden Schicht bestätigt, welche durch eine sterile, olivgrüne Sandschicht von der unteren Schicht getrennt ist.

\section{Jüngerer Löß II a}

Bei "La Adam" hat sich infolge der durch den teilweisen Einsturz der Decke gebildeten und hier bereits erwähnten Ơffnung der Jüngere Löß II a in einer ununterbrochenen Schicht in der ganzen Höhle abgelagert.

Der Einsturz bildet eine sterile, sehr dicke (bis 1,20 m) Schicht, welche auf einem Spät-Moustier liegt und seinerseits zwei Früh-Aurignac-Niveaus trägt.

Die höchstens $0,50 \mathrm{~m}$ dicke und in einer Tiefe $\mathrm{zwischen} 1-1,80 \mathrm{~m}$ befindliche Lößschicht bedeckt die Früh-Aurignac-Niveaus und enthält eine Fauna, in welcher die arktischen Tiere (Alopex lagopus, Rangifer tarandus, Ovibos sp.?) hier erstmals die maximale Häufigkeit erreichen. Eine gesteigerte Häufigkeit zeigen auch die Steppenformen, die durch Saiga tatarica, Bison priscus, Vulpes corsac, Putorius eversmanni und die Equiden (Equus przewalski, Equus (Asinus) bydruntinus, Equus caballus) vertreten sind. Die erwähnten Arten charakterisieren zusammen mit der in großer Zahl vorkommenden Crocuta spelaea das zweite Stadial in der Dobrudscha. Das Mammut und wollhaarige Nashorn verschwinden, und der Höhlenbär wird sehr selten. Neben diesen erhalten sich noch aus dem Früh-Aurignac in kleiner Anzahl Cervus elaphus und Megaceros giganteus.

Der Feuerherd auf dieser Schicht hat uns eine menschliche Industrie erschlossen, welche dem Mittel-Aurignac angehört. Unter den Steinwerkzeugen fallen Klingen, Schaber auf Klingenspitzen und ein gekielter Schaber auf, der eine pyramidenartige Form hat. Dieser Schicht kann man auch eine zweiseitige Spitze zuschreiben, die auf beiden Seiten unregelmäßig behauen ist. Die Grundkante ist abgerundet und die Längskanten sind gebogen. Dieses Steinwerkzeug gehört zur Szeleta-Technik. Das übrigens sehr verschiedene Rohmaterial, welches für die Erzeugung der Werkzeuge gedient hat, ist von guter Qualität und wurde importiert.

Die Lößablagerung in Stärke von $1,30 \mathrm{~m}$ in der „Adăpostul Rîndunelelor“ gehört ganz zum Mittel-Aurignac, was aus der Fauna hervorgeht, die durch Alopex lagopus, Saiga tatarica, Equus (Asinus) bydruntinus und Crocuta spelaea vertreten ist. Der Feuerherd dieses Horizontes hat eine kleine Anzahl von bearbeiteten Steinwerkzeugen geliefert, die teilweise atypisch sind, sich aber doch in die Technik des Mittel-Aurignac einreihen.

\section{Jüngerer Löß II b}

Dieser Löß, in einer maximalen Stärke von $0,80 \mathrm{~m}$, wurde in der Ablagerung auf einer Terrasse vor dem Eingang zur Höhle "La Adam" festgestellt, ferner im Eingang und auf einem kleinen Streifen im Innern der Höhle. Er liegt auf dem Prägravette und unter einem mächtigen, sterilen Einsturz, welcher im Innern der Höhle eine Stärke von $1,10 \mathrm{~m}$ erreicht.

Die Fauna wird durch eine zweite Häufigkeitsstufe der arktischen Tiere (Alopex lagopus und Rangifer tarandus) charakterisiert, ferner durch die Beständigkeit der Steppenformen wie Saiga tatarica, Bison priscus und Vulpes corsac. Ursus spelaeus und Crocuta spelaea erscheinen in starker Verminderung und sind im Aussterben, während Bos primigenius vollkommen fehlt.

Die Hirsche (Cervus elaphus und Megaceros giganteus) sind seltener, aber in der unten liegenden Prägravette-Schicht gut vertreten; in ähnlicher Weise verhalten sich die Equiden. 
Zum erstenmal erscheinen in dieser Ablagerung Schafe von kleiner Gestalt (Ovis sp.); und hier hat man auch die ersten Überreste des Homo sapiens fossilis gefunden.

Die Steinwerkzeuge, die auf dem Feuerherd dieses Horizontes im Eingang zur Höhle entdeckt wurden, zeigen eine deutliche Neigung zu kleiner werdenden Abmessungen; Klingen mit abgedrücktem Rücken sind - obwohl anwesend - doch ziemlich selten.

Die Ablagerung des Lösses dauert auch nach dem Mittelwürm in der Dobrudscha an, $\mathrm{da}$ er sich in den mächtigen Kalksteintrümmern des Post-Gravette und auch im Oberen Mesolithikum (Tardenois) befindet, welcher die untere (Hamangia) und die mittlere (Gumelniţa) neolithische Schicht trägt.

Tabelle 1

Die Gliederung des jüngeren Lösses in der Dobrudscha

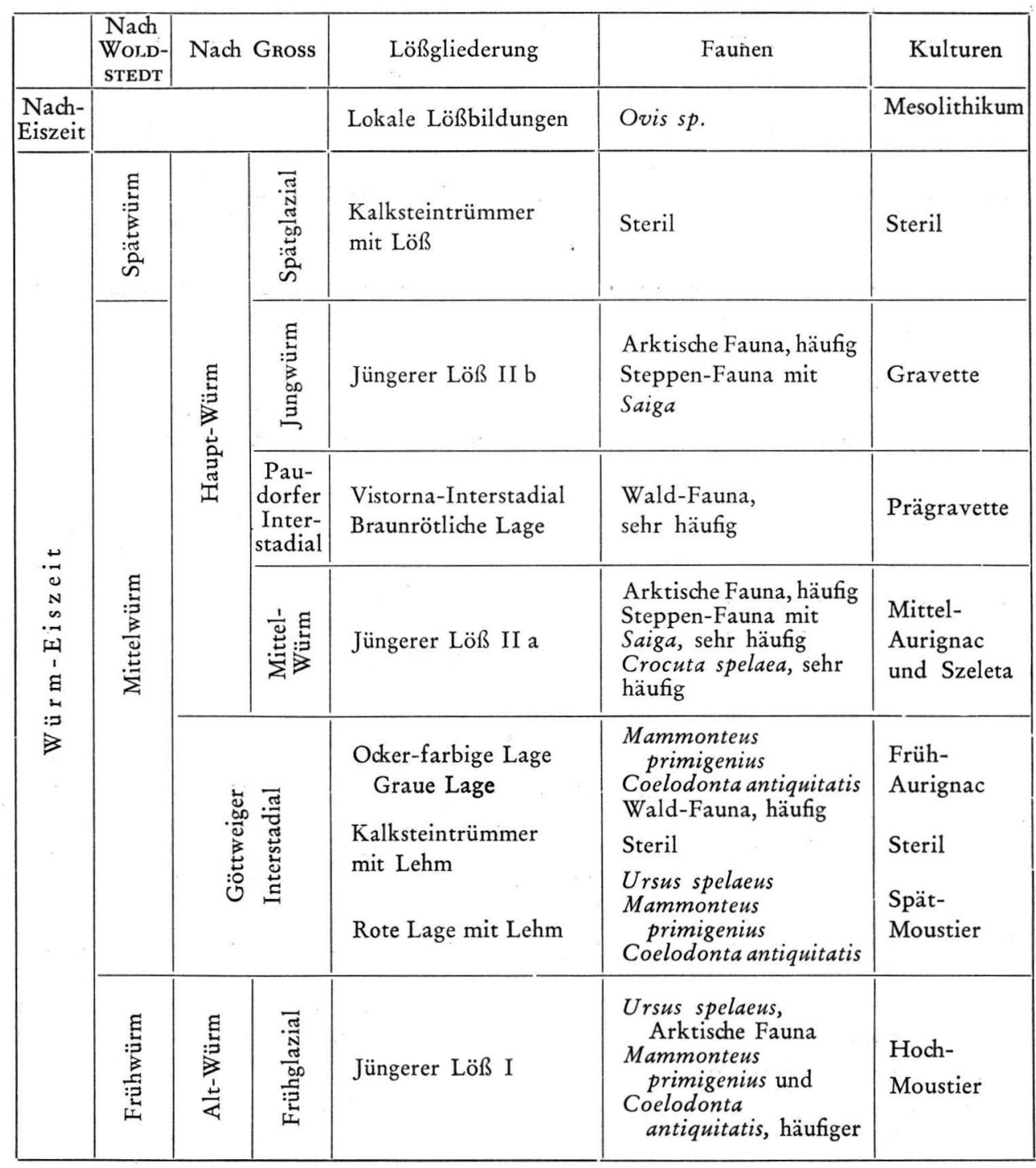




\section{Klima}

Für die Beurteilung der klimatischen Zustände, welche in der Dobrudscha in den Perioden der Lößbildungen geherrscht haben, bietet uns die Fauna die notwendigen Anhaltspunkte.

Die arktischen Tiere, vertreten durch Alopex lagopus und Rangifer tarandus sind aus dem Hoch-Moustier bekannt und erreichen zwei Häufigkeitsstufen: eine im MittelAurignac, zu welcher Zeit auch Ovibos erscheint, und die zweite im Gravette.

Es ist wegen des im Vergleich zu den anderen Arten seltenen Auftretens der arktischen Tiere sehr wahrscheinlich, daß diese in die Dobrudscha nur während der Winterwanderung gelangt sind. Für diese Behauptung spricht die Tatsache, daß die fossilen Uberreste des Renntiers nur Individuen angehören, welche älter als drei Monate waren.

Als Vertreter der eigentlichen Trockensteppe ist die Saiga-Antilope nicht nur in den Lößschichten, sondern auch in der gesamten Ablagerung vorzufinden und zeigt eine Steigerung der Häufigkeit im Mittel-Aurignac, ähnlich wie das bei der arktischen Fauna der Fall war. Es scheint, daß Saiga in dieser Gegend schon in der Interglazialzeit Riß/Würm vorhanden war und sich erst am Ende des Pleistozäns gegen Norden und Osten verzogen hat.

Die Hirsche bleiben, obwohl sie in den Perioden des Vorstoßes der arktischen Fauna selten werden, während des ganzen oberen Pleistozäns noch weiter bestehen und beweisen dadurch, daß das Klima in der Dobrudscha niemals außerordentlich streng war. Diese Tatsache erklärt auch die Anwesenheit der Ophidien, Lacertilien und Amphibien.

Bei der Deutung der Schwankungen in der Fauna gelangen wir zu der Schlußfolgerung, daß Mittel-Aurignac und Gravette zwei kältere klimatische Perioden waren.

Während der ganzen Würmzeit beherrschte die Waldsteppe die Landschaft der Dobrudscha, mit aufeinanderfolgendem Vorstoß und Rückzug des Waldes oder der Steppe. Es ist sehr wahrscheinlich, daß die Steppe im Mittel-Aurignac ihre größte Ausdehnung erreicht hat.

Auf Grund der angegebenen Daten können wir annehmen, daß in der Dobrudscha während des Oberen Pleistozäns sich "Jüngerer Löß“ in zwei hauptsächlichen Phasen abgelagert hat:

Jüngerer Löß I im Frühwürm bei gleichzeitigem Erscheinen der Hoch-Moustier-Kultur und Jüngerer Löß II im Mittelwürm in zwei Etappen: Jüngerer Löß II a in Verbindung mit dem Mittel-Aurignac und der Szeleta-Kultur und Jüngerer Löß II b mit der GravetteKultur. Wir müssen aber bemerken, daß die beiden Lagen des Jüngeren Lösses II durch ein Interstadial (Vistorna-Interstadia l) ${ }^{2}$ ) getrennt sind, welches dieser Gegend eigen ist und durch eine gemäßigte Fauna und eine Prägravette-Kultur gekennzeichnet ist.

Es ist das erste Mal, daß dieses Interstadial bei uns hervorgehoben wird. Die vorhergegangenen Studien haben nur den "Jüngeren Löß II“ erfaßt, ohne einen Unterschied zwischen Löß II a und II b zu machen. Diese Unterscheidung war in der Dobrudscha nur dank besonderer Bedingungen bei der Ablagerung möglich.

Es scheint, daß sich die Ablagerung des Lösses während des Spätwürms fortgesetzt hat.

\section{Bibliographie}

Botez. I. G.: Date paleolitice pentru stratigrafia loessului în Nordul Basarabiei. - Acad. Rom. Mem. Sect. St., Bucureşti 1930. - - Recherches de Paléontologie Humaine au Nord de la Bessarabie - Iaşi 1931.

Dumithescu, M., Ghika, M., Rădulescu, C., Samson, P. \& Terzea, E.: La Grotte „La Adam“ Station Pléistocène supérieure (im Druck).

2) Vistorna nennt man das Tal, in dem sich die Höhle „La Adam“ befindet. 
Ghika, M. \& Samson, P. (in Dumitrescu, M. etc.): L'Etude des Perissodactyles et des Proboscidiens.

Gross, H.: Das Göttweiger Interstadial - ein zweiter Leithorizont der letzten Vereisung. - Eiszeitalter und Gegenwart 7, S. 87-101, 1956.

Moroşan, N. N.: Le Pléistocène et le Paléolithique de la Roumanie du Nord-Est. - An. Inst. Geol. Rom. 19, Bucureşti 1938.

Rădulescu, C. (in Dumitrescu, M. etc.): L'Etude des Artiodactyles.

Radulescu, C., GHika, M. \& SAMson, P.: "Bordeiul de Piatră“ Staţiune mousteriana superioară. Mitteilungen bei der wissenschaftlichen Session des Institutes für Speologie "E. Racovita“, București, 10. - 12. Mai 1958.

Terzea, E. \& Samson, P. (in Dumitrescu, M. etc.): L'Etude des Carnivores.

Woldstedt, P.: Über die Gliederung der Würm-Eiszeit und die Stellung der Lösse in ihr. - Eiszeitalter und Gegenwart 7, S. 78-86, 1956. - - Das Eiszeitalter, Bd. II. Stuttgart 1958.

Manuskr. eingeg. 20. 7. 1959.

Anschrift d. Verf.: Dr. Petre Samson und Dr. Constantin Rădulescu, Speologisches Institut „E. Racoviţa“, Strada Dr. Capşa 8 - R. Lenin, Bukarest, Rumänien. 